Bull. Mater. Sci., Vol. 22, No. 2, April 1999, pp. 133-137. (C) Indian Academy of Sciences.

\title{
Design and fabrication of liquid phase epitaxy system
}

\author{
R VENKATARAGHAVAN, N K UDAYASHANKAR, BLASIUS VICTOR RODRIGUES, \\ K S R K RAO and H L BHAT* \\ Department of Physics, Indian Institute of Science, Bangalore 560012 , India
}

MS received 12 January 1999

\begin{abstract}
The design and fabrication of a simple and versatile liquid phase epitaxial (LPE) system has been described. The present LPE system makes use of the horizontal multi-bin boat and slider arrangement which enables the growth of even multilayered structures. The growth chamber is heated by a single-zone resistive furnace precisely controlled through a Eurotherm 902P temperature programmer and controller. The vacuum manifolds and accessories are set up in such a way as to ensure high vacuum needed for growth experiments. The provision is also made to admit high purity gases like hydrogen or nitrogen into the growth chamber. The design has been kept simple without sacrificing the versatility and adaptability for novel growth experiments. The typical films grown by this LPE system are also presented.
\end{abstract}

Keywords. Llquid phase epitaxy; multi-bin boat; indium antimonide; surface morphology.

\section{Introduction}

The liquid phase epitaxy (LPE) has been considered to be a simple and versatile technique for growing epitaxial films of III-V, II-VI and IV-IV compound semiconductors for material investigations and device applications (Panish et al 1970; Hsieh et al 1976; Liu et al 1977; Holmes and Kamath 1980; Astles 1990; Golube et al 1995; Ejeckman et al 1997). The development of GaAs and AlGaAs laser diodes is illustrative of how the LPE technique lends itself to the fabrication of optically useful devices based upon III-V binaries and ternaries (Kuphal 1991). A wide variety of such mixed crystals of differing energy gaps, suitable for microwave and optoelectronic devices have been grown using this technique. In particular, recent developments in antimony based III-V semiconductors (Kumagawa et al 1973; Srivastava et al 1986; Martinelli and Zamerowski 1990; Choi and Eglash 1991; Menna et al 1991) have prompted us to grow the epitaxial layers of these compounds for which purpose an indigenous LPE system was designed and fabricated in our laboratory. We present here the complete details of instrumentation and fabrication of LPE system which can be suitably adapted for trying out even heteroepitaxial structures.

\section{Instrumentation}

Basically, LPE systems with multi-bin boat and slider arrangement are variations on Nelson's theme of contacting the saturated melt with a substrate followed by

*Author for correspondence proper cooling to grow the epilayer (Nelson 1963). The doping profile or thickness control is done by terminating the growth by separating the melt and growing epilayer using various mechanisms. A plethora of methods have been devised for achieving this sequence (Dawson 1972; Astles 1990). The equipment required for LPE is relatively inexpensive. Compared to vapour phase epitaxy, the LPE technique is inherently safer, because the raw materials and waste products are less toxic. Therefore, one requires neither a big air conditioning system nor a complicated gas supply system (except for hydrogen or nitrogen) as in VPE or MOVPE. Figure 1 shows the schematics of the fabricated LPE system. For the purpose of better elucidation, the present LPE system can be broadly classified into the following subsystems which are described separately.

- The growth chamber

- The boat and slider system

- The vacuum manifolds and accessories

- The vacuum system

- The gas input line

- The furnace and temperature control system

\subsection{The growth chamber}

The growth chamber consists of a quartz reactor tube, in which are placed the growth crucibles. As can be seen from figure 1, the boat and slider are accessed from outside the growth system by the help of the retainer-tube and the slider-rod. The retainer-tube holds the boat in place during the entire growth procedure and enters the growth chamber from the side flange through 
a vacuum tight wilson seal. The retainer-tube is inserted halfway into the lower portion of the boat and terminates just under the growth bin. It also carries a thermocouple and hence serves in monitoring the temperature at the growth bin. The slider-rod runs parallel to the retainer-tube and it enters the growth chamber through a wilson seal and the end is hooked on to the slider, such that any movement of the slider-rod externally translates into the motion of the slider in the boat. The main reactor tube is a semiconductor grade quartz tube $35 \mathrm{~mm}$ ID and $38.1 \mathrm{~mm} \mathrm{OD}$, sealed on one end and the other end leading to a $\mathbf{T}$ flange with locknut to anchor it to the vacuum system and will be discussed in $\S 2.3$. The retainer-tube and the slider-rod are made from quartz with $6 \mathrm{~mm}$ and $4 \mathrm{~mm} O D$, respectively. The slider-rod is bent at the end to hook into the boat slider.

\subsection{The boat and slider system}

As already mentioned, this LPE system makes use of the horizontal multi-bin-slider arrangement, which is almost universally used for the growth of heterostructures like laser diodes. Standard design considerations have been followed in the design of the boat and slider (Kuphal 1991). Although other variants of the basic boat-slider system do exist in practice, the present design is among the simplest, economically viable and quite efficient for material investigations and research. The growth-boat consists of a substrate holder with the substrate held in the recess of the slider (see figure 2) and containing differently composed solutions in its bins. As the solutions generally do not stick to purified graphite, they can be wiped off the substrate to terminate growth. It is also possible that when the solution is pushed over the substrate, the latter might float upwards because its specific density is less than that of the solution. Therefore care has been taken to see that the width of the solution chambers perpendicular to the pushing direction is smaller than the substrate width. Another crucial point in a sliding boat is the separation between the substrate surface and the walls of the slit. If this slit is too large, solution is carried over into the following chamber, and the composition of subsequent solutions is altered. Solution carry-over not only depends on this, but also on the solution height and the pull-speed. If the slit is too small, the graphite or crystalline particles will cause scratches on the surface of the grown layer in the sliding direction. The problem becomes even more severe when thick layers with considerable edge growth are deposited. In present system we have selected the slit height to vary in the range between $15 \mu \mathrm{m}$ and $100 \mu \mathrm{m}$. It may also be noted that, the drawback of the sliding technique is the relative motion of graphite parts causing abrasion by particles, which can lead to growth defects. Particle abrasion was, however, almost suppressed by the use of high-density, high quality graphite, supplied by BARC, Mumbai and machined in the Department Workshop.

\subsection{The vacuum manifolds and accessories}

The vacuum manifold consists mainly of (see figure 1): (a) $\mathrm{T}$ joint with locknut, (b) $\mathrm{S} / \mathrm{S}$ straight tube, (c) $\mathrm{T}$

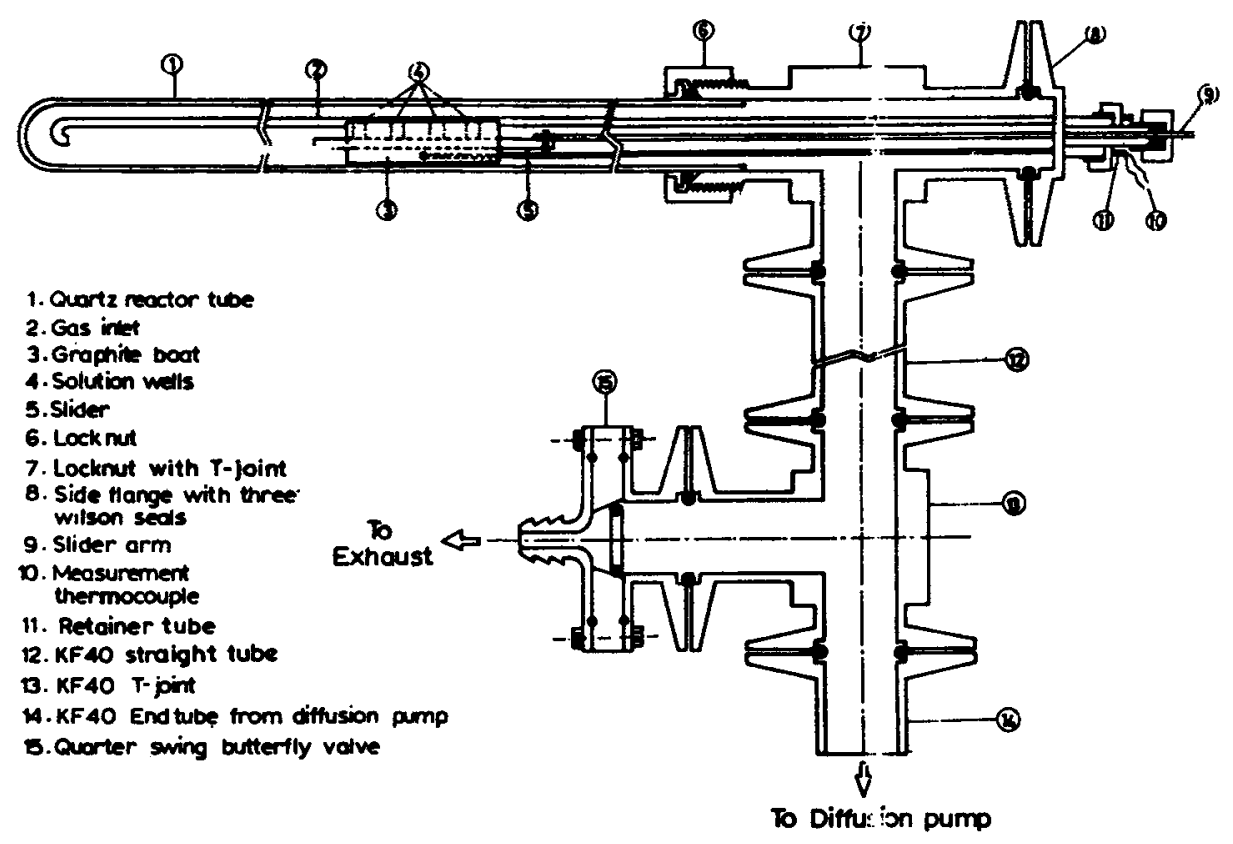

Figure 1. Schematics of LPE system showing vacuum manifolds and other accessories. 
joint and (d) quarter swing butterfly valve. All vacuum manifolds are made from S/S 304 and are electropolished as required for a clean vacuum system to avoid degassing etc. All these joints end in standard KF 40 couplings so that the two parts may be effectively and quickly coupled through a O-ring holder and a quick clamp. All connecting $\mathrm{O}$-rings are made of viton, which are preferred to neoprene O-rings. The KF 40 coupling has been effectively used as it offers $28 \%$ O-ring compression as against $15 \%$ for other Wilson seals. Only at the quartz tube-manifold joint a locknut coupling has been used. A S/S mesh is used at the O-ring holder between the locknut $T$ and the straight tube to avoid any foreign particle that may accidentally fall into the vacuum pump. The lower end of the straight tube couples to a $T$ joint which bifurcates the reactor pathway to the diffusion pump and the exhaust line. A quarter swing butterfly valve available at the side of the lower $T$ joint, controls opening and closing of the exhaust line and is accessible on the front panel of the system through a push-pull rod. The exhaust end of the valve ends in a nozzle connected through plastic tubing and leads out to the atmosphere through a needle valve NV2 (also on the front panel) to the atmosphere.

\subsection{The vacuum system}

The entire growth chamber, gas flow lines and exhaust lines are evacuated through a vacuum pumping system consisting of a oil vapour diffusion pump, backed by a compatible rotary pump. The high vacuum chamber of the vacuum system terminates in a coliar supplied with
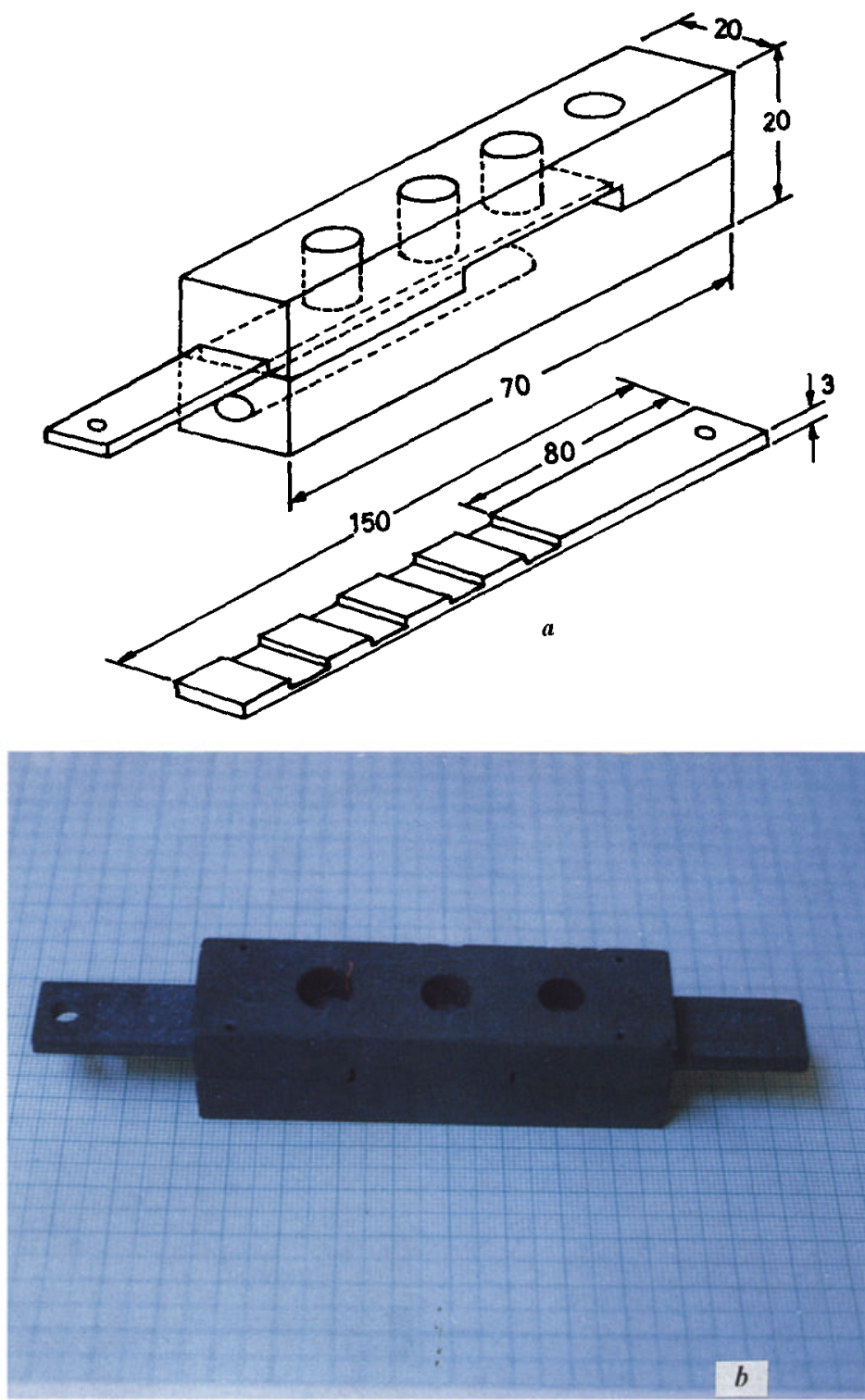

Figure 2. Horizontal multi-bin boat and slider arrangement: (a) schematic and (b) photograph 
a KF 40 coupling. This directly couples to the bottom of the $T$ joint at the end of the vacuum line from the reactor. The standard vacuum system configuration is used with valves for roughing, backing and high vacuum valve made available on the front panel. The vacuum at the reactor is measured through a set of pirani and penning gauge system and is again in the standard vacuum system configuration.

\subsection{The gas input line}

High purity gases enter the growth chamber through a $3 \mathrm{~mm}$ ID quartz tube at the side flange of the locknut $\mathrm{T}$ and the tube runs across the length of the reactor tube and the gas effuse at its closed end. The input of the gas into the quartz tube is controlled through a standard gas cylinder pressure regulator and a needle valve NV1. The output of the pirani gauge meter can be taken on to external Digital Multimeter (DMM) through a phonojack at the pirani meter panel. The voltage thus monitored in $\mathrm{mV}$ gives a measure of the pressure in the system.

\subsection{The furnace, travel and temperature control} system

The furnace to be used in this system was designed and fabricated in the laboratory. The external dimensions of the furnace is a cylinder of diameter $30 \mathrm{~cm}$ and a length of $50 \mathrm{~cm}$. The furnace is supported on a moving flat platform cradled by curved MS flats, welded to the trolley mainframe. The wheels offer 1 inch track width, sliding on support rails made of 1 inch square tubes. The axle width was kept at $25 \mathrm{~cm}$ for stability. The railing allowed a total travel length of $1 \mathrm{~m}$ and a 0.25 inch undercut in the wheel on the innerside offered self stopping of the carriage at the end of the travel guide. The LPE furnace, coaxial to the reactor tube had a bore of $50 \mathrm{~mm}$, capable of maintaining up to $1000^{\circ} \mathrm{C}$ at the centre. The muffle, made from recrystallized alumina, $50 \mathrm{~mm} \mathrm{ID} \times 52 \mathrm{~cm}$ long, was wound with $16 \mathrm{SWG}$ Kanthal A1 resistive element. The coil was wrapped in ceramic wool blanket, after covering the entire length with furnace cement. A stainless steel coaxial cylinder $49 \mathrm{~cm}$ in length and $16 \mathrm{~cm}$ in diameter, was placed midway between the muffle and furnace wall to serve as a reflector to contain excessive radiation losses. The volume between the reflector and the furnace wall was filled with loose ceramic fibres. Of the total length of $52 \mathrm{~cm}$ of the muffle, $18 \mathrm{~cm}$ of the muffle was closed from one end with quartz blanket. The binding wires were brought out through the furnace with ceramic beads for insulation and were connected to a bakelite post at the side wall of the furnace. The total resistance of the furnace was found to be $16 \Omega$, with a power up of
$0.5 \mathrm{KW}$ for a temperature of $1000^{\circ} \mathrm{C}$. A type $\mathrm{K}$ chromelalumel thermocouple pair was introduced along the muffle wall so as to be at the centre, to record the maximum achievable coil temperature.

The furnace is controlled through a Eurotherm 902P temperature programmer and its associated power module (SCR). The 902P is a sixteen segment controller capable of stability better than $0.1{ }^{\circ} \mathrm{C}$ for a compatible furnace and ramps as low as $0.1{ }^{\circ} \mathrm{C}$ per day. The furnace has an optimal $10 \mathrm{~cm}$ isothermal region (at $400 \mathrm{~W}$ ) and quite sufficient for the present boat dimensions. Figure 3 shows the photograph of the fabricated LPE system.

\section{Typical results}

Employing the above LPE system, epilayers of InSb and InTISb have been grown. The initial optimization experiments were carried out to obtain $\mathrm{InSb}$ homoepitaxial layers. The smooth and continuous films were obtained at a growth temperature of $425^{\circ} \mathrm{C}$ (see figure 4a). However, in applications where electrical isolation is necessary, it is important to be able to grow InSb films on substrates of high resistivity. Therefore an attempt has been made to grow InSb on (100) GaAs substrates with a lattice mismatch of $\sim 15 \%$. Imposing stringent growth conditions, we were able to obtain good quality heteroepitaxial InSb films (figure 4b). Recent reports have predicted the alloying of InSb and $\mathrm{TlSb}$ which will serve as useful detector material in the long wavelength IR (LWIR). Also, other reports of MOCVD growth of InTISb have encouraged the thought that InTISb is probably a futuristic replacement for $\mathrm{HgCdTe}$ (Choi et al 1991, 1994). In view of this, InTISb films were grown on GaAs using the similar set of growth parameters as of InSb/GaAs. The surface morphology of one such film is shown in figure $4 \mathrm{c}$. As can be seen from the figure, the film is reasonably smooth except for a pit at the

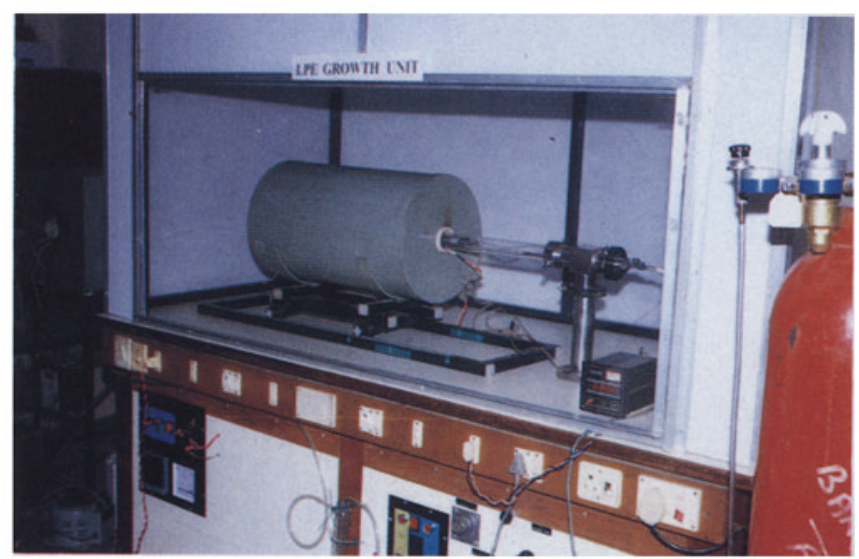

Figure 3. Photograph of the LPE growth unit. 

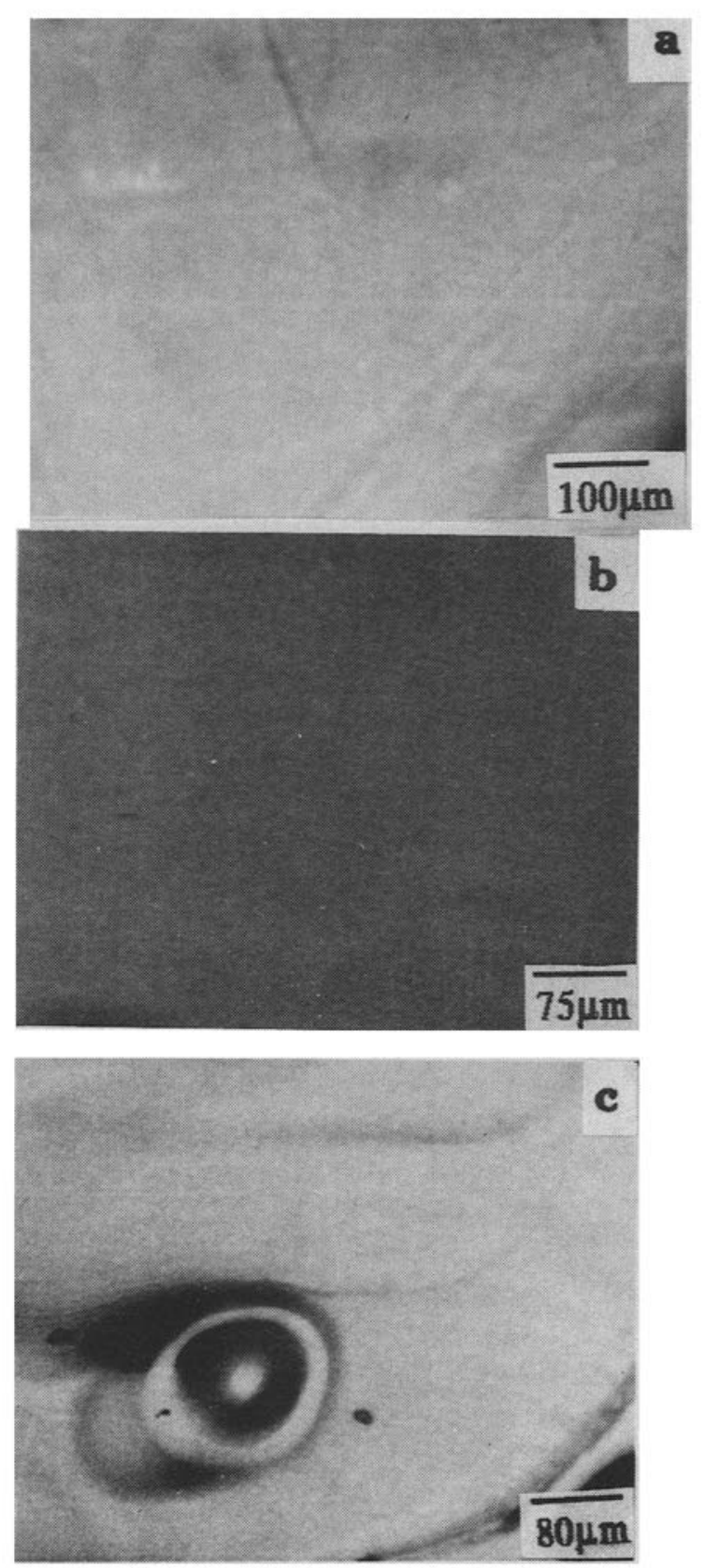

Figure 4. Micrographs showing surface morphology of (a) homoepitaxial layer of InSb (thickness $-22 \mu \mathrm{m}$ ), (b) InSb/GaAs film (thickness $-28 \mu \mathrm{m}$ ) and (c) InTISb layer on GaAs (thickness $\sim 25 \mu \mathrm{m})$.

centre. The thickness of the film as determined using a point probe was found to be $\sim 25 \mu \mathrm{m}$. It was grown at a temperature of $425^{\circ} \mathrm{C}$ by imposing a supersaturation $(\Delta T)$ of $7.5^{\circ} \mathrm{C}$. For the ternary epilayers $\mathrm{X}$-ray diffraction pattern confirms the formation of InTISb phase and IR transmission spectrum shows the shift in the detection limit to LWIR. The details of LPE growth of the above structures have been communicated elsewhere (Udayashankar et al 1999).

\section{Conclusions}

In this paper, we have described the design and fabrication of an indigenous LPE system which can be used for materials investigation and basic research. Employing this system, certain Sb-based III-V semiconductors were grown. The design has been kept simple without sacrificing the flexibility and versatility and can be suitably adapted for novel growth experiments.

\section{Acknowledgements}

The authors would like to thank the financial support of ISRO-IISc Space Technology Cell for this work. One of the authors (NKU) gratefully acknowledges the financial support obtained through Quality Improvement Programme (QIP) of Ministry of Human Resource Development, New Delhi.

\section{References}

Astles M G 1990 in Liquid-phase epitaxial growth of III-V compound semiconductors (Bristol: IOP. Pub. Ltd)

Choi H K and Eglash S J 1991 IEEE J. Quantum Electron. QE-27 1555

Choi Y H, Besikci C, Sudharsanan R and Razeghi M 1991 Appl. Phys. Lett. 63361

Choi Y H, Staveteig P T, Bigan E and Razeghi M $1994 \mathrm{~J}$. Appl. Phys. 756

Dawson L R 1972 in Progress in solid state chemistry (Oxford: Pergamon Press)

Ejeckman F E, Seaford M L, Lo Y H, Hou H Q and Hammons B E 1997 Appl. Phys. Lett. 71776

Golube L V, Egorov A V, Novikov S V and Shmartsev Yu V 1995 J. Cryst. Growth 146277

Holmes D E and Kamath G S 1980 J. Electronic Mater. 995

Hsieh J J, Rossi J A and Donelly J P 1976 Appl. Phys. Lett. 28709

Kumagawa M, Witt A F, Lichtensteiger M and Gatos H C 1973 J. Electrochem. Soc. 12058

Kuphal E 1991 Appl. Phys. A52 380

Liu W K, Winesett J, Ma W, Zhang X, Santosh M B, Fang X N and McCann J 1977 J. Appl. Phys. 811708

Martinelli R U and Zamerowski T 1990 Appl. Phys. Lett. 56 125

Menna R J, Capewell D R, Martinelli R U, York P K and Enstrom R E 1991 Appl. Phys. Lett. 592127

Nelson H 1963 RCA Rev. 24603

Panish M B, Hayashi I and Sumuski S 1970 Appl. Phys. Lett. 16326

Srivastava A K, DeWinter J C, Caneau C, Pollack M A and Zyskind J L 1986 Appl. Phys. Lett. 48903

Udayashankar $\mathbf{N} K$, Venkataraghavan $R$, Nirmal Kumar and Bhat H L 1999 Phys. Status Solidi (communicated) 